\title{
Die unerwünschte Arzneimittelwirkung Schmerz
}

\author{
Birgit Böhmdorfer, Daniel Schaffarzick, Marietta Nagano, Susanne Melitta Janowitz, \\ Ekkehard Schweitzer
}

Eingegangen: 31. Dezember 2011 / Angenommen: 21. Juni 2012 / Online publiziert: 18. Juli 2012

(c) Die Autor(en) 2012. Dieser Artikel ist auf Springerlink.com mit Open Access verfügbar.

\section{Pain as adverse drug reaction}

Summary We present a multidisciplinary (anaesthesiology_clinical pharmacy_bioinformatics) analysis of pain as possible adverse drug reaction taking different manifestations of pain, indication groups, relevance to the Austrian drug market and possible mechanistic influence of drugs on development and apprehension of pain into consideration.

We designed an overview that shows how transmitters that play a part in nociception and antinociception can be influenced by drugs. This allows conclusions to the dolorigene potential of therapeutics.

Keywords: Pain, Adverse drug reaction, Pharmacist

Zusammenfassung Wir analysieren interdisziplinär (Anästhesie - klinische Pharmazie - Bioinformatik) Schmerz als mögliche unerwünschte Arzneimittelwirkung. Wir berücksichtigen dabei die Aspekte unterschiedlicher Schmerzentitäten, Indikationsgruppen, Relevanz der Substanz im österreichischen Arzneimittelmarkt und möglichen mechanistischen Einfluss der Arzneimittel auf Schmerzentstehung und -Wahrnehmung.

Mag. ${ }^{a}$ pharm. B. Böhmdorfer, aHPh $(\bowtie) \cdot$ Mag. ${ }^{a}$ pharm. M. Nagano . Mag. ${ }^{\text {a }}$ pharm. S. M. Janowitz

Anstaltsapotheke, Krankenhaus Hietzing mit Neurologischem Zentrum Rosenhügel, Wolkersbergenstrasse 1,

1130 Wien, Österreich

E-Mail: birgit.boehmdorfer@wienkav.at

Mag. ${ }^{a}$ B. Böhmdorfer

Institut für Allgemein-, Familien und Präventionsmedizin, Paracelsus Medizinische Privatuniversität, Salzburg, Österreich

DI (FH) D. Schaffarzick

Duller Engineering GmbH, Salzburg, Österreich

Dr. med. E. Schweitzer

Abteilung für Anästhesie und Intensivmedizin, interdisziplinäre Schmerzambulanz, Krankenhaus Hietzing mit Neurologischem Zentrum Rosenhügel, Wien, Österreich
Wir haben eine Tabelle erstellt, die Transmitter, die an der (Anti)-Nozizeption beteiligt sind und deren mögliche Beeinflussung durch Pharmaka darstellt. Dadurch ist ein Rückschluss vom Wirkmechanismus von Arzneimitteln auf deren dolorigenes Potential ursächlich mechanistisch möglich.

Schlüsselwörter: Schmerz, Unerwünschte Arzneimittelwirkung, Apotheker, Medication Appropriateness

\section{Einleitung}

Pharmakotherapie findet im Spannungsfeld zwischen therapeutischem Nutzen und möglichen unerwünschten Nebenwirkungen statt [1-4].

Unsere Zielsetzung war, aufzuzeigen, wie groß der quantitative Aspekt der möglichen Nebenwirkung Schmerz im Hinblick auf unterschiedliche Indikationsgruppen und Anzahl der in Frage kommenden Substanzen ist. Zwar wird in der Literatur zum Thema das Nebenwirkungsprofil Schmerz durchaus im Hinblick auf einzelne Substanzgruppen, wie z. B. den Statinen [5] und Myopathien oder neuropathischen Schmerz im Zusammenhang mit bestimmten Zytostatika [6] oder unter dem einzelnen klinischen Aspekt des Kopfschmerzes [7] diskutiert, aber nicht in der in diesem Artikel präsentierten Vollständigkeit der Schmerzentitäten und unter Einbeziehung aller am österreichischen Markt vorhandenen Arzneimittelgruppen.

\section{Material und Methoden}

Es wurde eine computerunterstütze Recherche in der Datenbank „Clinical Pharmacology“ [8] durch ,adverse reactions reports" mit der Verschlagwortung "*pain", "*pathy" und "*ache" sowie eine Kategorisierung der erhaltenen Substanznamen nach verschlagworteter Schmerzentität, Indikationsgruppe und Verfügbarkeit der Substanz als ein in Österreich zur humanmedizini- 
schen Verwendung registriertes Arzneimittel (Quelle: Medis IntranetKH basierend auf der Austria Codex Fachinformation 2009/2010, Österreichische Apotheker-Verlagsgesellschaft 2009 [9]) durchgeführt. Wenn Substanzen in mehreren Schmerzrubriken aufschienen, wurden sie dort mit jeweils einer Nennung berücksichtigt. Die Datenbank Clinical Pharmacology [8] liefert neben Einzelsubstanzen auch Substanzkombinationen als mögliche Treffer. Substanzkombinationen wurden nur in begründeten Ausnahmen als solche vermerkt, z. B., wenn es zum Zeitpunkt der Recherche weltweit kein Monopräparat der mutmaßlich schmerzassoziierten Einzelkomponente gab (Nennung Index Nominum [10]), ansonsten wurden ausschließlich Einzelsubstanzen in die Analyse aufgenommen.

Diese Substanzen wurden einer ersten Datenaufbereitung anhand der Schmerzrubrik, in die sie durch die Datenbank verschlagwortet wurden unterzogen.

Im zweiten Analysenschritt wurde der Überlegung Rechnung getragen, dass bei Substanzen, die aufgrund ihres Wirkmechanismus' bevorzugt in der Therapie von Schmerzen eingesetzt werden, aufgrund dieser Indikation eine eindeutige Zuordnung dieser Arzneimittel als auslösende Agentien des Schmerzgeschehens schwierig bis unmöglich ist. Die Gruppe der nicht-steroidalen Antirheumatika und der opioiden Analgetika wurde daher aus der weiteren Analyse ausgeschlossen. Ebenso wurde mit der Gruppe der nicht in Österreich in Präparaten zur Anwendung im humanmedizinischen Bereich registrierten Substanzen verfahren, da sich unsere Überlegungen auf den österreichischen Raum konzentrieren.

Um einem mechanistisch-ursachenorientierten Ansatz Rechnung zu tragen, erstellten wir eine Liste mit am Schmerzgeschehen und der - wahrnehmung beteiligten Transmitter, sowie deren mögliche Beeinflussung durch Arzneimittel.

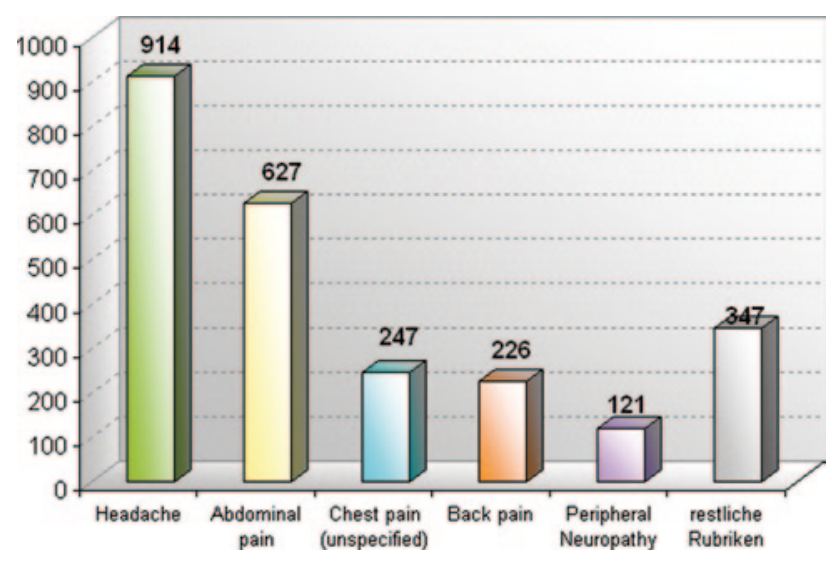

Abb. 1 Ergebnis der Zuordnung zu 21 Schmerzgruppen (gesamt 2.581 Nennungen) die Bezeichnungen der x-Achse entsprechen der Nomenklatur der konsultierten, englischsprachigen Datenbank

\section{Ergebnisse}

Die erste Analyse ergab 2.581 Treffer aus gesamt 21 Schmerzrubriken. Die Gruppen, die die höchste Anzahl an Substanznennungen enthielt, waren „Headache“ (Kopfschmerz) und „Abdominal pain“ (Bauchschmerzen), siehe Abb. 1.

Durch Ausschluss der Substanzen aus der Gruppe der nicht-steroidalen Antirheumatika und der opioiden Analgetika reduzierte sich die Trefferanzahl auf 2.482.

Eine weitere Einschränkung auf die Arzneistoffe, die in Österreich zum Zeitpunkt der Recherche registrierten Präparaten zur humanmedizinischen Anwendung führte zu 1.713 Nennungen. Diese wurden nach Indikationsgruppen dargestellt, siehe Abb. 2.

Die größte Anzahl an Nennungen (321 von 1.713) entfiel dabei auf die Gruppe der Antiinfektiva.

Die Analyse der Transmitter und deren Bedeutung für die Schmerzwahrnehmung bzw. deren Beeinflussung durch Medikation führte zu den Ergebnissen, die in der Tab. 1 dargestellt werden: Viele Substanzen, die
Abb. 2 Einteilung nach Indikationsgruppen (Gesamt 1.713 Nennungen, nach Ausschluss der Substanzen aus der Gruppe der nichtsteroidalen Antirheumatika und der opioiden Analgetika sowie Substanzen, die zum Zeitpunkt der Recherche in Österreich nicht zur humanmedizinischen Anwendung registriert waren)

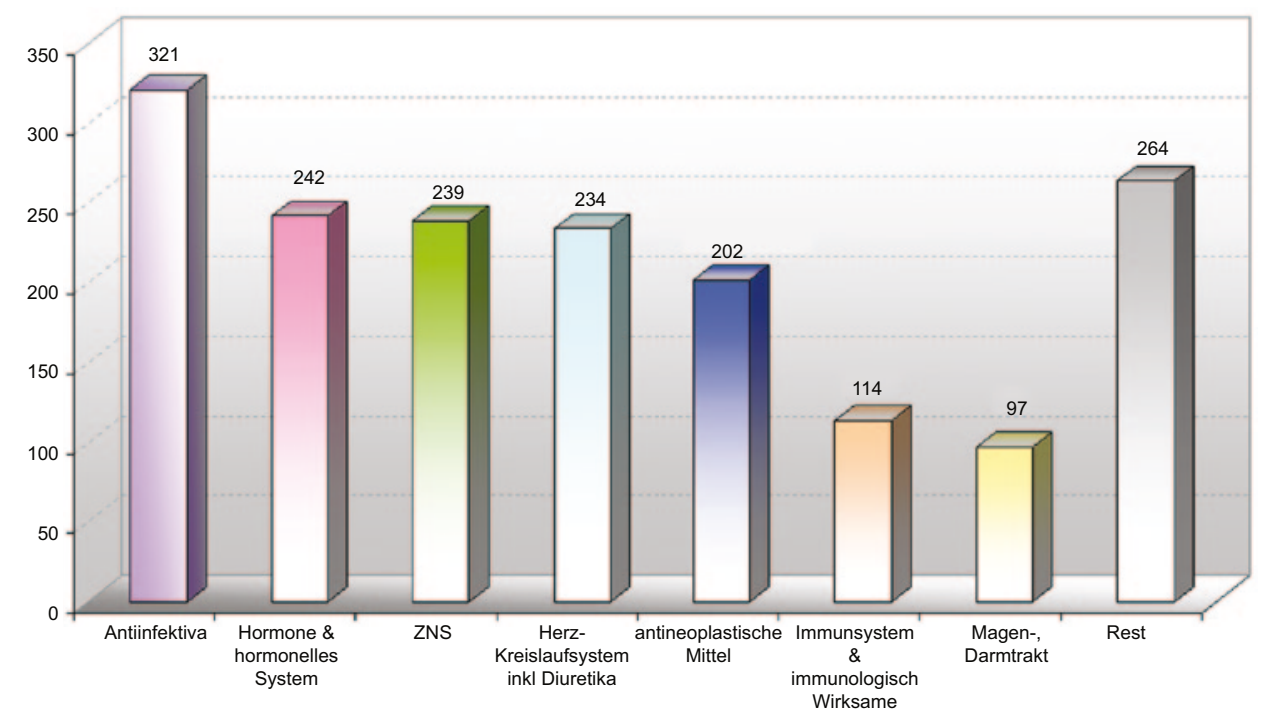




Tab. 1. Transmitter (in Auswahl), die an der (Anti)-Nozizep-
tion beteiligt sind und deren mögliche Beeinflussung durch
Pharmaka (in Auswahl)

5-HT Serotonin, ACEAngiotensin-Converting-Enzyme, BK Bradykinin, Gaba Gamma-Amino-Buttersäure, NA Noradrenalin, NO Stickstoffmonoxid, NSAR nicht-steroidale Antirheumatika, PG Prostaglandine, SNRI Serotonin-Noradrenalin-Reuptake-Inhibitor, SSRI selektiver Serotonin-Reuptake-Inhibitor ${ }^{a}$ Endorphine: beta-Endorphin, die Dynorphine, Methionin und Leucin-Enkephalin

zur medikamentösen Therapie eingesetzt werden können auf die komplexen Transmissionskaskaden, die die körperlichen Ereignisse von Schmerz und/oder Entzündung begleiten Einfluss nehmen [11, 12]. Wir schlagen die Verwendung des Terminus' „dolorigen“ für potentiell schmerzauslösende Arzneimittel vor.

\section{Diskussion}

Die Anzahl der Substanznennungen und die Diversität der Rubriken an Schmerzkategorien die wir bei unserer Analyse gefunden haben veranschaulichen, dass es sich beim Thema Schmerz als Nebenwirkung um ein komplexes Phänomen handelt.

Unsere Recherche zeichnet sich durch folgende Aspekte aus:

Die uns bekannte Literatur zum Thema berücksichtigt zumeist exklusiv spezielle Einzelaspekte von Schmerz als Nebenwirkung wie z. B. ausschließlich Myopathien oder neuropathischen Schmerz. Wir hingegen berücksichtigen Schmerz als unerwünschte Arzneimittelwirkung unabhängig von der Art des Schmerzes.

Wir berücksichtigen Schmerz als unerwünschte Arzneimittelwirkung unter besonderer Beachtung der tatsächlichen Relevanz am österreichischen Arzneimittelmarkt, indem wir uns auf Substanzen konzentriert haben, die in in Österreich aus zugelassenen Arzneispezialitäten am Markt sind.

Wir haben eine Tabelle erstellt, die Transmitter, die an der (Anti)-Nozizeption beteiligt sind und deren mögliche Beeinflussung durch Pharmaka darstellt. Dadurch ist ein Rückschluss vom Wirkmechanismus von Arzneimittel auf dessen dolorigenes Potential ursächlich mechanistisch möglich.

Wir ziehen aus unseren Überlegungen folgenden Schluss:

Die Fülle an potentiell schmerzauslösenden Substanzen und die Diversität der Rubriken an Schmerzkategorien veranschaulichen, dass es sich beim Thema Schmerz als unerwünschte Arzneimittelwirkung um ein relevantes und komplexes klinisches Phänomen handelt. Da die Wahrnehmung von Schmerz allerdings ein rein subjektives Erleben des einzelnen Patienten ist, ist es schwierig bis unmöglich, diese einer objektiven Quantifizierung von außen zu unterziehen. Es kommt daher zwangsläufig zu unterschiedlichen Wahrnehmungen und damit auch Beurteilungen durch behandelnden Arzt und Patienten.

In der Literatur ist bspw. beschrieben, dass beim Auftreten von Myopathien unter Statintherapie die behandelnde Ärzte und ihre Patienten die Häufigkeit des Auftretens der unerwünschte Arzneimittelwirkung Schmerz deutlich unterschiedlich beurteilen [13]. Hier könnte die Einführung des "self-reportings" von unerwünschten Arzneimittelwirkungen durch die Patienten selbst, wie sie in einigen Ländern üblich ist [14] mithelfen, ein bisher tendenziell unterrepräsentiertes Phänomen besser abzubilden und damit zukünftig berücksichtigen zu können.

Unsere Überlegungen haben die Stärke, eine komplexe Problematik systematisch aufzuarbeiten. Uns ist keine vergleichbare Analyse bekannt, die sich so wie wir allen Schmerzentitäten und allen relevanten Substanzen, die derzeit in Österreich therapeutisch eingesetzt werden gleichermaßen widmet und daher gleicherma- 
ßen geeignet ist, das Ausmaß und die Relevanz des Themas zu veranschaulichen.

Unsere Arbeit erfährt eine Limitation durch die pauschale Exklusion der nicht-steroidalen Antirheumatika und opioiden Analgetika aus der Analyse. Daraus könnte der durchaus diskutable Rückschluss gezogen werden, dass diese Indikationsgruppen per definitionem als nicht-dolorigen zu betrachten sind.

Weiters wäre es interessant, die Anzahl der Nennungen innerhalb einer Indikationsgruppe ihrer quantitativen therapeutischen Bedeutung gegenüberzustellen. Beispielsweise scheint die Gruppe der „Herz-Kreislaufmedikamente inkl. Diuretika“, die eine überproportional häufig eingesetzte Relevanz und eine Unzahl an Vertretern aufweist, mit 234 Nennungen auf, während die auf einem deutlich kleineren Patientenkollektiv angewandte Gruppe der „Antineoplastika“, deren Anzahl an therapeutisch in Österreich eingesetzten Einzelsubstanzen vergleichsweise überschaubar ist, 202 mal in Zusammenhang mit der Nebenwirkung „Schmerz" aufscheint. Eine derartige Analyse müsste allerdings Verbrauchsdaten berücksichtigen.

\section{Danksagung}

Wir danken den Vortragenden des Kurses „scientific publishing“ der PMU Salzburg, Dr. Tobias Kiesslich und Doz. Dr. Hans-Christian Bauer für die Hilfestellung im Rahmen des Erarbeitens eigener Texte zur Publikation.

BB dankt Mag. Ulrike Muster für eine umsichtige und vorausschauende Apothekenleitung, in der eigene Ideen und Forschung Raum finden.

\section{Interessenkonflikt}

Alle Autoren erklären keine Firmenaffilationen im Hinblick auf die Erstellung dieses Artikels. Es besteht kein Interessenkonflikt.

Open Access Dieser Artikel unterliegt den Bedingungen der Creative Commons Attribution License. Dadurch sind die Nutzung, Verteilung und Reproduktion erlaubt, sofern der/die Originalautor/en und Quelle angegeben sind.

\section{Literatur}

1. Schuler J, Duckelmann C, Beindl W, Prinz E, Michalski T, Pichler M. Polypharmacy and inappropriate prescribing in elderly internal-medicine patients in Austria. Wiener klinische Wochenschrift. 2008;120(23-24):733-41.
2. Hanlon JT, Schmader KE, Samsa GP, Weinberger M, Uttech $\mathrm{KM}$, Lewis IK, et al. A method for assessing drug therapy appropriateness. J Clin Epidemiol. 1992;45(10):1045-51.

3. Onder G, Petrovic M, Tangiisuran B, Meinardi MC, Markito-Notenboom WP, Somers A, et al. Development and validation of a score to assess risk of adverse drug reactions among in-hospital patients 65 years or older: the GerontoNet ADR risk score. Arch Intern Med. 2010;170(13):1142-8.

4. Schneider EL, Campese VM. Adverse drug responses: an increasing threat to the well-being of older patients: comment on „Development and validation of a score to assess risk of adverse drug reactions among in-hospital patients 65 years or older“. Arch Intern Med. 2010;170(13):1148-9.

5. Cham S, Evans MA, Denenberg JO, Golomb BA. Statin-associated muscle-related adverse effects: a case series of 354 patients. Pharmacotherapy. 2010;30(6):541-53.

6. Xiao WH, Bennett GJ. Chemotherapy-evoked neuropathic pain: abnormal spontaneous discharge in A-fiber and C-fiber primary afferent neurons and its suppression by acetylL-carnitine. Pain. 2008;135(3):262-70.

7. Headache Classification Subcommittee of the International Headache Society. The international classification of headache disorders: 2. Aufl. Cephalalgia. 2004;24(Suppl. 1):9-160.

8. Clinical Pharmacology (database on the Internet). http:// www.clinicalpharmacology.com (2010). Zugegriffen: 5 . Nov. 2010.

9. Medis IntranetKH (database on the Internet). http:// medis.wienkav.at/frameset9163.htm (2010). Zugegriffen: Nov. 2010.

10. Schweizer Apothekerverband. Index Nominum International Drug Directory. Stuttgart: Medpharm GmbH Scientific Publishers; 2004.

11. Libert F, Bonnefont J, Bourinet E, Doucet E, Alloui A, Hamon $\mathrm{M}$, et al. Acetaminophen: a central analgesic drug that involves a spinal tropisetron-sensitive, non-5-HT(3) receptor-mediated effect. Mol Pharmacol. 2004;66(3):728-34.

12. Mutschler E. Arzneimittelwirkungen: Lehrbuch der Pharmakologie und Toxikologie, 9. Aufl. Stuttgart: Wiss. Verlagsgesellschaft; 2008.

13. Golomb BA, McGraw JJ, Evans MA, Dimsdale JE. Physician response to patient reports of adverse drug effects: implications for patient-targeted adverse effect surveillance. Drug Saf. 2007;30(8):669-75.

14. Blenkinsopp A, Wilkie P, Wang M, Routledge PA. Patient reporting of suspected adverse drug reactions: a review of published literature and international experience. Br J Clin Pharmacol. 2007;63(2):148-56. 